\section{Automated data acquisition for multidimensional scaling analysis}

\section{ELIZABETH AIKEN and DAVID I. MOSTOFSKY Boston University, Boston, Massachusetts}

Multidimensional scaling (MDS) techniques offer rational alternative strategies in cases in which a mapping of cognitive or perceptual processes is desired. Despite the mathematical complexities that underlie these procedures, the experimenter is relieved of much of the computation labor by the numerous computer scaling programs available and several articulate monographs that introduce the novice to the necessary procedural and conceptual phases (Kruskal \& Wish, 1978; Schiffman, Reynolds, \& Young, 1981). This report describes a program designed to assist the experimenter in the data collection phase, which is often the most time-consuming phase. This is so for several reasons: A well-designed protocol will commonly specify that the subject make a response on a judgmental scale to indicate the similaritydissimilarity estimate between each of $\mathrm{N}(\mathrm{N}-1) / 2$ items such that (1) a line of fixed length be available for making the response and (2) each item pair be presented without opportunity for comparison with prior or subsequent items in the series. Preparation of materials can be extremely time-consuming, and the scoring of the responses followed by organizing the data into appropriate matrix form for computer analysis can be an even greater unpleasantry.

In the program described here, the subject first is given instructions on the CRT and then is given the sample items. The test items are then presented with instructions to the subject to position an " $\mathrm{x}$ " along the line of "same-different" that corresponds to his/her judgment of item similarity. At the end of the experiment, two data matrices are computed for the subject: (1) item-pair distances, where distance is departure from maximum similarity (in inches) and (2) item-pair response times (in seconds). Response latency is measured from the first opportunity that the subject has to position an " $x . "$ These triangular matrices are stored for later processing by ALSCAL or similar routines.

Description. This program incorporates structured programming techniques by using one main routine that calls several subroutines. The MAIN program controls program flow. LDMEAS loads up the measuring array with distance measurements that correspond to each " $x$ " that can be made on the 5-in. line-each " $\mathrm{x}$ " is .1389 in. wide. INSTRN presents the subject with instructions and practice sessions. READIN reads in the

The authors are affiliated with the Department of Psychology, Boston University, Boston, MA 02215. actual stimuli. INIT initializes the array that keeps track of which combinations of stimuli have been presented. PROCES processes all possible combinations of stimuli and presents the comparisons in random order. The randomizing algorithm used is selection with replacement. If many data items are to be used, it is efficient to use a sampling-without-replacement algorithm. RESPON displays the line and measures the subject's responses. WRITMS writes the distance-measurement matrix, and WRITTM writes the response-time matrix. TIME is a system function that returns times in hours, minutes, seconds, and one-hundredths of a second. Most operating systems provide a built-in time function, but if you are using a computer without this feature, a time function could be simulated through looping. RAN is also a system function that returns random numbers between a specified range. CLEAR is an IBM assembler macro that clears the screen of an IBM 3278 terminal between presentations of stimuli. On another IBM system this macro would be fine, but in a non-IBM environment it will be necessary to know if a character can be written to the screen to clear it or an assembler macro must be called.

Input. Input to the program consists of: (1) an identification code for the data file being used, and the number of stimuli, both entered interactively by the experimenter, (2) input data file consisting of a list of individual stimuli, which can be a maximum of 20 characters (the first eight stimuli are for the practice session), and (3) variable input from the subject in the form of a typed " $x$ " under the 5-in. line indicating the subject's perception. The IBM 3278 terminals allow for 80 characters to be interactively read.

Output. During the program run, instructions are first presented to the screen if the subject desires. The combinations of stimuli and the 5-in. line are presented one per screen. Final output of half-filled matrices are written to two output files, on recording distance measurements and one recording response times. Instructions and output are easily modified to fit the user's need.

Technical Information. This program was created, tested, and used on an IBM 3081 with OS/VS system on an IBM 3278-3 terminal. The program is written in WATFIV but is compatible with any standard FORTRAN with a few minor changes. We are aware that moving the " $x$ " along the length of the line from its leftmost origin may introduce a response bias, particularly for estimates of decision times. This problem may be effectively solved by replacing the standard keyboard response with a joystick response and making the appropriate program alterations. Similarly, there are several system dependencies that have been previously acknowledged. The time, random, and clear functions will require modification for use on systems other than IBM. 
The object code requires 14,344 bytes of storage. Array storage requires 3,788 bytes. Compile time is $.08 \mathrm{sec}$, and execution time depends on the number of stimuli presented and the subject's response time.

Availability. A listing of the program and all the system subroutines with documentation may be obtained free of charge from David Mostofsky, Department of Psy. chology, Boston University, 64 Cummington Street, Boston, MA 02215.

\section{REFERENCES}

Kruskal, J. B., \& Wish, M. (1978). Multidimensional scaling. Beverly Hills, CA: Sage.

Schiffman, S., ReYnolds, M. L., \& Young, F. W. (1981). Introduction to multidimensional scaling. New York: Academic Press.

(Revision accepted for publication March 8, 1984.) 